\title{
Thermal Considerations of a Power Converter with Components Embedded in Printed Circuit Boards
}

\author{
Rémy Caillaud ${ }^{1}$, Cyril Buttay ${ }^{1}$, Roberto Mrad ${ }^{2}$, Johan Le Leslé ${ }^{2}$, Florent Morel ${ }^{1}$, Nicolas Degrenne ${ }^{2}$ \\ and Stefan MOLLOV ${ }^{2}$ \\ ${ }^{1}$ Université de Lyon, INSA de Lyon, CNRS, École Centrale de Lyon, Université Claude Bernard Lyon 1, Ampère, \\ F-69621, France \\ ${ }^{2}$ Mitsubishi Electric R\&D Centre Europe, 1 Allée de Beaulieu, 35708 Rennes, France
}

\begin{abstract}
Printed-Circuit-Board (PCB) technology is attractive for power electronic systems as it offers a low manufacturing cost for mass production. Integration technologies such as device embedding have been developed to take advantage of the interlayer space in multi-layer PCBs and to increase the performances (Electrical, Thermal). However, the PCB technology offers limited power dissipation due to the low thermal conductivity $(\approx 0.3 \mathrm{~W} /(\mathrm{m} \cdot \mathrm{K}))$ of its composite substrate.

In this paper, we consider PCB embedding for a $3.3 \mathrm{~kW}$ AC/DC bidirectional converter. We describe the integration of not only the power dies, but also the gate drive circuits and the power inductor, with a special focus on the thermal management. The manufacturing processes of the boards are presented. Two thermal models based on finite elements (FE) of this converter stage are introduced. The accuracy of these models is validated against experiments. The results show that a simplified FE model offers satisfying accuracy and fast simulation, even considering the relatively complex structure and layout of the PCBs.
\end{abstract}

\section{INTRODUCTION}

$\mathbf{O}$ VER THE LAST DECADES, evolution in power electronics has mainly been driven by improvements in the switching components. Since 2001, semiconductor devices made out of wide band-gap materials have been commercially introduced: first Silicon Carbide ( $\mathrm{SiC}$ ) and more recently Gallium Nitride $(\mathrm{GaN})$ [1]. However, their performances are currently limited by their packaging. Nowadays, the next driver for progress is considered to be better integration and packaging technologies $[2,3]$. In particular, future packaging technologies should offer better electrical and thermal performances and low cost.

Among the possible integration technologies, PrintedCircuit Board (PCB) is especially attractive: because it is a mature technology, many advanced design tools are available, as well as a complete supply chain. It allows a high density of interconnects, and uses relatively inexpensive materials. Many manufacturing steps of a PCB rely on batch processes, allowing a large number of interconnects to be processed at once. Its main limitation is the low thermal conductivity of its base material (most commonly a glass-epoxy composite). Even the best materials have thermal conductivities lower than $10 \mathrm{~W} /(\mathrm{m} \cdot \mathrm{K})[4]$, a fraction of that achieved by the ceramic materials used in power electronics (e.g. $150 \mathrm{~W} /(\mathrm{m} \cdot \mathrm{K})$ for AlN). As a consequence, multilayer PCBs are mainly used for low-power converters $(<100 \mathrm{~W})$. For higher power levels,

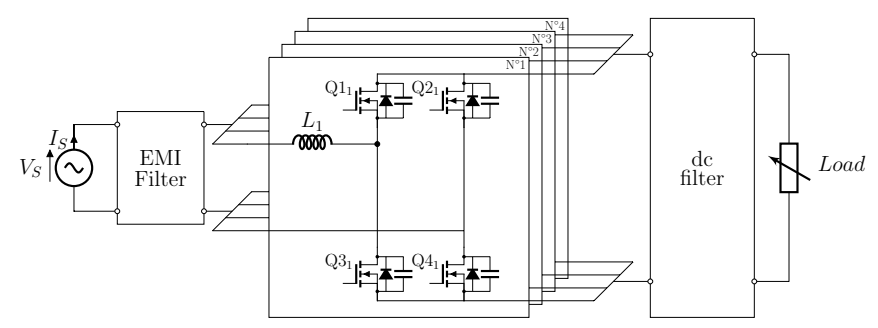

Figure 1: Circuit diagram of the converter. The topology includes an EMI filter, a Power Factor Corrector (PFC) and a dc filtering stage. The PFC uses 4 interleaved cells $[15,16]$.

Insulated Metal Substrates (a single-layer, thin PCB bonded onto a thick metal plate) or ceramic substrates are preferred.

With the ever increasing need for more compact electronic systems, integration technologies have been developed to take advantage of the inter-layer space in multi-layer PCBs by embedding components in the substrate [5]. Embedding the devices in the PCB allows to overcome some of the thermal limitations (by reducing the thickness of low-conductivity composite between the components and the thermal management system). Some signal electronic systems were produced with almost all components embedded in PCB [6], but for power electronics, the research has mainly been focused on embedding the active devices only $[7,8]$. The embedding of passives has also been investigated for low power converter (1-100 W) with the embedding of Surface Mount Technology (SMT) components (Resistors, Capacitors, ICs) [9], of magnetic cores $[10,11]$, or of formed components using capacitive or resistive layers [12]. It is worth noting that aside from the scale, this vertical stacking of flat structures (also called "2.5D") is very close to the "chip stacking" concept. Chip stacking is commonly used in microelectronics, and the thermal modeling of such structures have been explored in papers such as [13] or [14]. However, these advanced models require a considerable implementation effort.

In this paper, we investigate the embedding of all components of an AC/DC converter (semiconductor power devices, corresponding gate driver, and inductor), rated at $3.3 \mathrm{~kW}$, with a special focus on thermal aspects. In section II, some details are given regarding the components to be embedded and the chosen physical implementation. The manufacturing process is described in section III. Then, in section IV, two thermal 


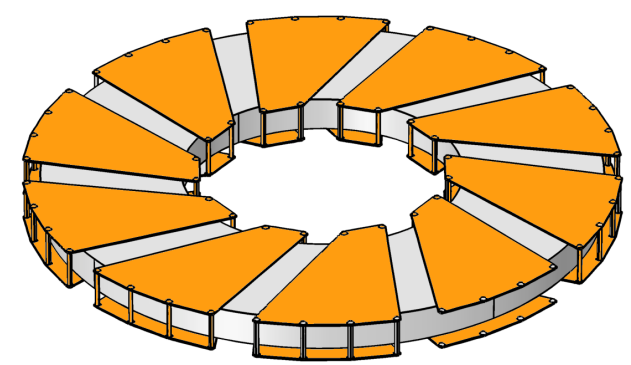

Figure 2: Drawing of an "Embedded Toroidal" inductor. The dielectric layers are not show.

models are presented, while the acquisition of experimental temperature data is presented in section V. Finally, the results are compared and discussed in section VI.

\section{DESIGN OF A PFC CELL}

The converter topology to be implemented is presented in Fig. 1. It includes an EMI filter, several interleaved Power Factor Corrector (PFC) cells and dc filtering stage. The components of this circuit have been selected using an optimization procedure to achieve the best power density/efficiency ratio $[15,16]$. The optimized circuit is composed of $4 \mathrm{PFC}$ cells (each handling a power of $825 \mathrm{~W}$, for a total of $3.3 \mathrm{~kW}$ ). In the remaining of the paper, we will focus on a single PFC cell, for which the input (ac) voltage is $240 \mathrm{~V}, 50 \mathrm{~Hz}$, the output (dc) is $400 \mathrm{~V}$ and the input current $4 \mathrm{~A}$ RMS.

A PFC cell is composed of 4 SiC MOSFETs (CPM2-12000080, Wolfspeed) in a full bridge configuration, driven by 2 half-bridge gate drive circuits (based on an IRS21864 IC, Infineon, with their peripheral components - resistors, capacitor, diodes in SMT package - ) and an inductor $(34.7 \mu \mathrm{H})$. The inductor uses an "Embedded Toroidal" structure which corresponds to an embedded annular magnetic core with a winding formed around by PCB tracks and vias (Fig. 2) [17]. Note that both half-bridge operate at different frequencies: in Fig. 1, Q1 and Q3 control the current in L1, and switch at high frequency $(180 \mathrm{kHz})$, while Q2 and Q4 are only turned-on or off with respect to the polarity on the mains (i.e. at $50 \mathrm{~Hz}$ ).

These 3 sets of elements (power semiconductor devices, gate drive circuit and inductor) are embedded in different PCBs which are stacked on top of a heatsink to form a compact assembly. Each PCB is separated by a layer of Thermal Interface Material (TIM). The TIM chosen is a thin (200 microm), electrically insulating (breakdown voltage $3000 \mathrm{~V}$ ) elastomeric material which is soft enough to conform to the PCB patterns (Bergquist Sil-Pad 1500ST). The boardto-board interconnections are done with wires soldered in metallized through holes. A schematic view of the assembly is given in Fig. 3, and a photograph of the exploded PFC cell is visible in Fig. 4.

With the chosen approach, the empty space created around each component is filled with resin, as compared to air in a standard converter. The replacement of air by resin allows a better conduction of the heat from the components to the heatsink [18] and allows easier interconnection. However, the

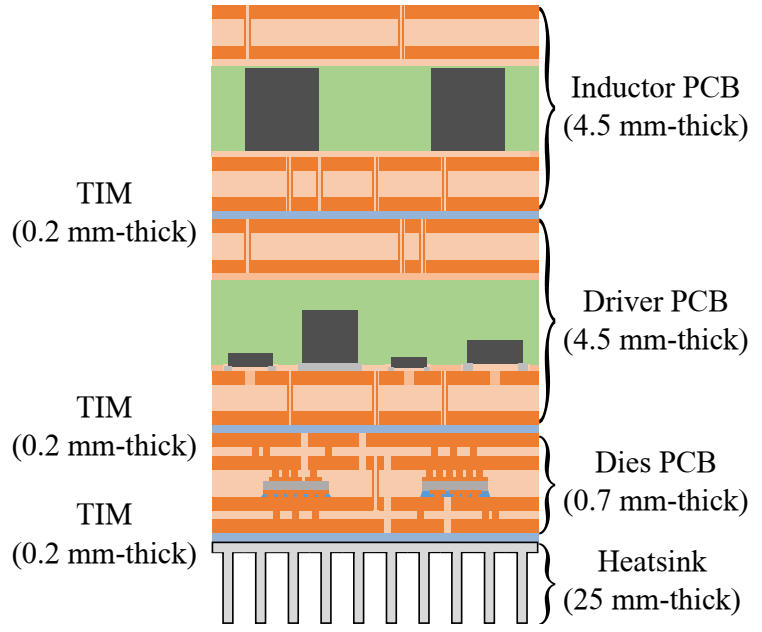

Figure 3: Cross section of a PFC cell. The PCBs thicknesses are exaggerated with regard to the heatsink thickness and of the width of the boards. The components and layout in the drawing are only illustrative..

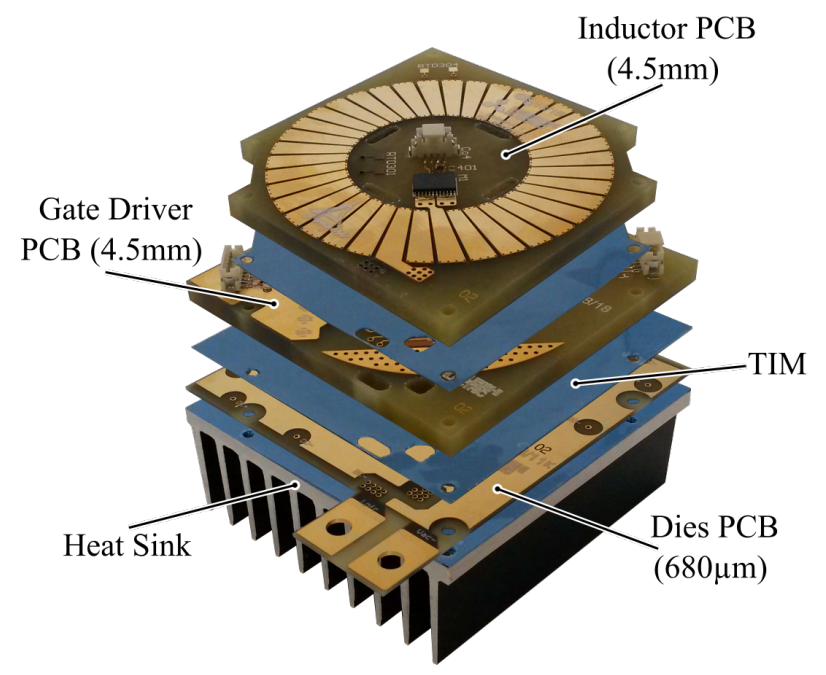

Figure 4: Exploded view of a PFC cell.

thermal conductivity of the FR-4 (Epoxy resin - fiberglass composite) which composes the substrate of the PCB remains low $(0.3 \mathrm{~W} /(\mathrm{m} \cdot \mathrm{K}))$. With the thick structure presented in Fig. 3 (more than $1 \mathrm{~cm}$ ), investigations are required to ensure no component will exceed its maximum operating temperature.

\section{MANUfacturing OF The BoARdS}

As depicted in Figs. 3 and 4, 3 boards are used to form the PFC cell. While two of them have similar structure ("inductor board" and "driver board", both $4.5 \mathrm{~mm}$-thick), this is not the case for the "Dies board", which is much thinner $(0.7 \mathrm{~mm})$. From a manufacturing point-of-view, this requires the production of two different panels, each with a different manufacturing process. For the "Dies board", a "thin panel" is processed, while the "inductor" and "driver" PCBs correspond to a "thick panel". Both of these panels have 4 copper layers (numbered 1 to 4 , top to bottom).

1) Thin Panel: Only bare $\mathrm{SiC}$ dies are embedded in the "Thin PCB" panel, between both inner layers. The process 

a)
b)

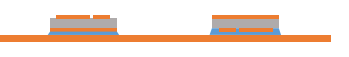
c)

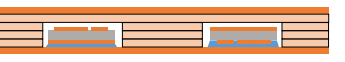
d)

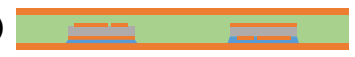
e)

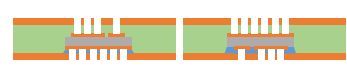
f)

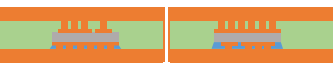
g)

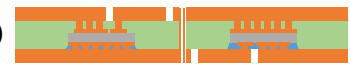
h)

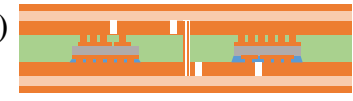
i)

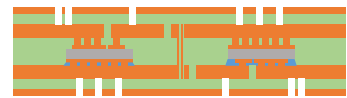
j)

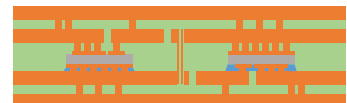
k)

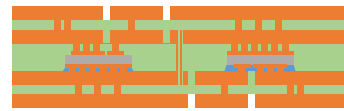
Copper etching

Figure 5: Manufacturing step for the panel with embedded dies: "Thin PCB". Dark orange denotes copper; light orange denotes prepreg, i.e. un-cured glassepoxy layers which are cured during the lamination process; green denotes cured glass-epoxy layers.

is presented in figure 5. It starts with a $35 \mu \mathrm{m}$ plain copper sheet (Fig. 5a) on which the dies are accurately positioned and bonded with non-conductive glue. Some dies are mounted "bottom up" (flip-chip, Fig. 5b) so that interconnects with fast voltage transient (i.e. the middle points of the full-bridge structure) are away from the heatsink, thus reducing commonmode noise. On the contrary, dc voltage potentials (the drain of Q1 and Q2, or the source of Q3 and Q4 in Fig. 1) are routed on large planes close to the heatsink to foster capacitive coupling to ground while providing an electrostatic screen between fast switching potentials and the heatsink.

Sheets of glass-fiber pre-impregnated with resin (so-called "prepregs") are then stacked to compensate for the die thickness (Fig. 5c) and to isolate the dies from the copper layer above. This stack is laminated (Fig. 5d) to form a solid part.

The dies interconnections ("track to dies" vias) are drilled on both sides using a laser ("microvias", Fig. 5e). The laser power is calibrated using sacrificial dies, so that the vias extend all the way through the insulating material $(25 \mu \mathrm{m}$ for the bottom glue layer and $50 \mu \mathrm{m}$ for the top laminated sheet) and stop

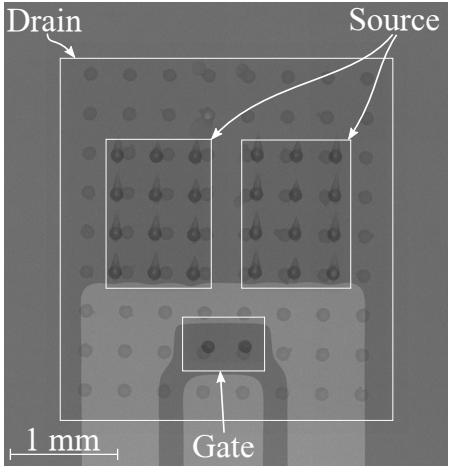

Figure 6: X-ray image of an embedded $\mathrm{SiC}$ die (white outline of the die and pads added for clarity), showing the microvias drilled on both sides.

when reaching the pads of the dies (finished with $8 \mu \mathrm{m}$ copper, a value recommended by the PCB manufacturer). Copper is then electro-plated to fill the vias (Fig. 5f). After the copper electro-deposition, the thickness of the copper layers is $70 \mu \mathrm{m}$. They are then etched to form the PCB tracks (Fig. 5g). The $\mathrm{X}$-ray image of an embedded die is visible in Fig. 6. The via positioning accuracy with respect to the dies is theoretically estimated at $100 \mu \mathrm{m}$ (practically, it was found to be $33 \mu \mathrm{m}$ in the worst case), thanks to accurate manufacturing equipment and monitoring of board deformation.

Finally, the external layers (layers 1 and 4) are added, using similar steps (Figs. $5 \mathrm{~h}$ to $5 \mathrm{k}$ ). These two layers are required so that the final panel is rigid enough for handling, has a symmetrical structure (to prevent bowing), and for routing the interconnects.

2) Thick Panel: For this panel, components are no longer glued. Soldering is used instead, as it is compatible with standard SMT packages. This requires to pattern the copper layer 3 before populating it with components. The process begins with a double-sided laminate with $70 \mu \mathrm{m}$ thick copper (Fig. 7a). Through-all vias are mechanically drilled (Fig. 7b) and electro-plated. The copper is then etched to form the circuit on both sides (Fig 7c). Some solder paste is deposited on the board by stencil-printing, with a pattern matching the footprint of the components. The components are placed on the paste and soldered using a reflow oven (Fig. 7d).

Two prepreg layers are then placed, with cut windows matching the components location and size (Fig. 7e). The aim of these prepreg layers is to provide adhesion for additional layers and to isolate the magnetic cores from copper layer 3. A spacer, composed of several PCB cores and prepregs layers is placed on top (Fig. 7f).

Finally (figs $7 \mathrm{~g}$ and $\mathrm{h}$ ), two more prepreg layers are placed on top to isolate the magnetic core from copper layer 2 and to bond the laminate forming the layers 1 and 2 (which was manufactured similarly to figs. 7 a to c). Through-all vias are then manufactured to interconnect all four layers.

\section{Thermal Models}

With the complex structure depicted in Fig. 3, it is important to ensure that all embedded components operate below their maximum temperature. Here, we present the thermal models 
a)

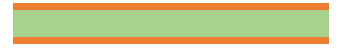

Thin laminate $(150 \mu \mathrm{m})$

b)

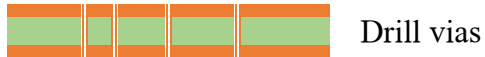

c)

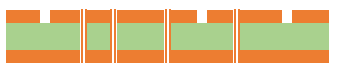

Copper electro-deposition Copper etching

d)

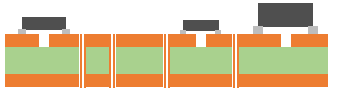

Place and solder SMT components

e)

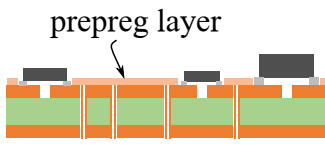

Place bonding layer

f)

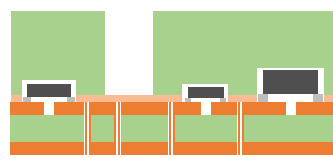

Place PCB core with machined cavities

g)

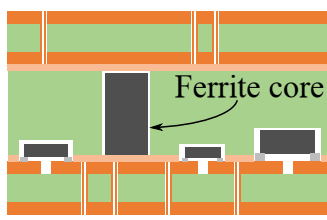

h)

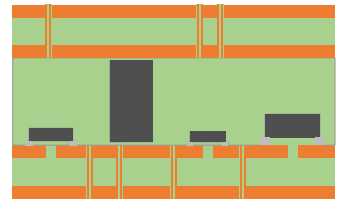

Laminate under pressure

Figure 7: Manufacturing step for the panel with both embedded SMT components and magnetic cores: "Thick PCB".

which are used to compute the temperature distribution in the cell. First, a simple model is presented for the convective heat transfer from the heatsink. In a second time, two models are proposed for the heat conduction in PCB stack, and are compared regarding their complexity and computation time.

\section{A. Heatsink Model}

While the heatsink used here is fairly simple (a piece of extruded aluminium with straight fins), its Finite Element Model (FEM) is relatively complex: not only does it require many elements to mesh the heatsink itself, but the surrounding air must also be meshed so Computational Fluid Dynamics (CFD) can be applied. This is very important as the air movement (convection) dictates the heat exchange between the heatsink (i.e. the heat flux between the converter) and its environment. To keep the thermal simulations of the converter simple, a two-step approach is used: first, a detailed heatsink model is built, and simulated in a variety of conditions; then a

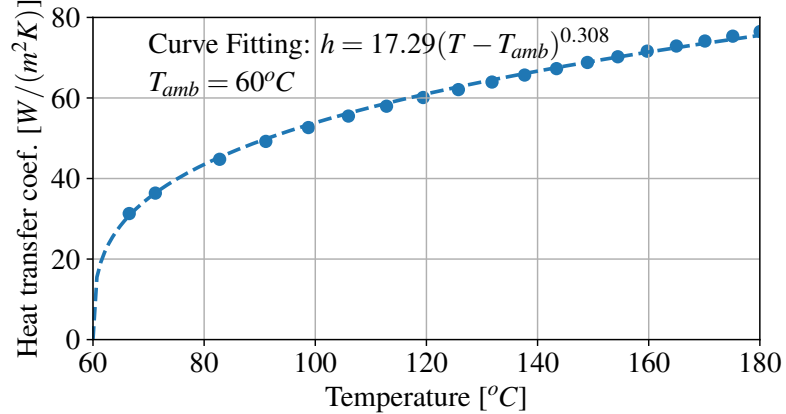

Figure 8: Simulation results of the heat transfer coefficient as a function of the temperature of the heatsink, considering convection and radiation effects, for an ambient temperature of $60^{\circ} \mathrm{C}$.

simple equation is fitted to the results of these simulations. For converter simulations, which are performed with a different FE simulator for practical reasons, this equation is used as a boundary condition, in place of the FE heatsink model.

The detailed heatsink geometry is drawn in 3D using the Icepack software (Ansys). The heatsink is placed inside a "cabinet", which represents a large volume of air around the heatsink. The cabinet dimensions need to be larger (twice, in our case) than the heatsink dimensions so its boundaries do not disturb the airflow. Air flow is only allowed to cross the top and bottom boundaries of the cabinet. Gravity is taken into account. In addition to convection phenomena, the simulator also calculates the heat exchanged by radiation. The ambient air temperature is $60^{\circ} \mathrm{C}$ (maximum ambient temperature specified in our application).

A uniform power dissipation is applied on top of the heatsink baseplate. The temperature of the heatsink (considered uniform) is the result of the simulation. Several simulations are run for power dissipation levels ranging from $1 \mathrm{~W}$ to $50 \mathrm{~W}$. The heatsink temperature is extracted for each simulation. It then becomes possible to calculate an equivalent heat transfer coefficient $h$ at baseplate-level such as:

$$
h=\frac{P}{A_{\text {base }} \cdot \Delta T}
$$

with $P$ the power dissipation, $A_{\text {base }}$ the top surface area of the baseplate and $\Delta T$ the temperature difference between the heatsink and the ambient air. The heat transfer coefficient as a function of the heatsink temperature is presented in Fig. 8.

Finally, an equation of the form $h=\alpha(\Delta T)^{\beta}$ is fitted to the data from Fig. 8. This equation is used in the PCB-stack model as a boundary condition for the converter area which is in contact with the heatsink. This way, CFD is no longer necessary in the thermal simulations of the converter. This approach, however, can only apply to steady-state conditions (it does not consider the "thermal mass" of the heatsink), and is only valid over the power/temperature domain for which it was identified.

\section{B. PCB-Stack Models}

To some extent, a model of the PCB-stack described in Fig. 3 is simple to build: the stack has flat external surfaces; 
Table I: Thermal conductivities of the different layers for the "Approximated Stack-up" thermal model.

\begin{tabular}{l|l}
\hline Layer Name & $\begin{array}{c}\text { Thermal Conductivity }(\mathrm{x}, \mathrm{y}, \mathrm{z}) \\
(\mathrm{W} /(\mathrm{m} \cdot \mathrm{K}))\end{array}$ \\
\hline \hline Copper Layer : Plane & $\lambda=(400,400,400)$ \\
\hline Copper Layer : PCB Traces & $\lambda=(0.3,0.3,400)$ \\
\hline Substrate layer : Standard & $\lambda=(0.3,0.3,0.3)$ \\
\hline Substrate layer : Vias & $\lambda=(0.3,0.3,50)$ \\
\hline TIM (Sil-Pad 1500 ST, Bergquist) & $\lambda=(1.8,1.8,1.8)$ \\
\hline Baseplate & $\lambda=(237,237,237)$ \\
\hline Ferrite & $\lambda=(5,5,5)$ \\
\hline
\end{tabular}

Table II: Losses in each power element of a PFC cell for the converter at nominal power (as estimated during the circuit design stage [15]).

\begin{tabular}{l|c}
\hline Dissipating element & Losses (W) \\
\hline \hline High Frequency Die (Per die, 2 dies per cell) & $5.83 \mathrm{~W}$ \\
\hline Low Frequency Die (Per die, 2 dies per cell) & $0.81 \mathrm{~W}$ \\
\hline Inductor (One inductor per cell) & $8.25 \mathrm{~W}$ \\
\hline
\end{tabular}

the side surfaces can be considered adiabatic, heat only being exchanged through the bottom (heatsink) and top surfaces. In this way, only heat conduction has to be considered, with heat exchange coefficients as boundary conditions on the top and bottom surfaces. Here, the complexity comes from the internal structure, which is an intricate pattern of parts with different heat conductivities and thicknesses. Overall, there are 12 copper layers in the stack, many copper vias through the substrate layers, and large parts of ferrite material.

The complete FEM model of such a structure would require a very fine mesh (with many elements measuring only a few microns). Two approaches are proposed below to generate models: the first only uses a coarse approximation of the copper layout, with the objective to offer acceptable results with a low computation time, while the second considers the actual copper layout (albeit with the finer features removed). Both models are built and simulated using COMSOL.

1) Approximated layout model: Here, each layer of the PCB stack is represented by a unique prismatic block, with an in-plane thermal conductivity set depending on the layout of that layer. Two cases are considered here: either a layer mainly comprises large copper planes, or it mainly contains thin tracks. Indeed, heat is going to spread much more over a plain copper layer (in this case, in-plane conductivity is considered to be that of copper, $400 \mathrm{~W} /(\mathrm{m} \mathrm{K}))$ than in the case of a copper layer with thin PCB tracks covering only a small part of the surface (in-plane conductivity set at $0.3 \mathrm{~W} /(\mathrm{m} \mathrm{K})$, that of the substrate). For the substrate layer, an area with a high concentration of copper vias will increase the thermal conductivity in the z-axis. This model has a relatively low number of elements (300 000).

The thermal conductivity of the substrate layer is $0.3 \mathrm{~W} /(\mathrm{m} \cdot \mathrm{K})$. For a substrate layer with a high concentration of vias, the $\mathrm{z}$-axis thermal conductivity is increased to

Table III: Temperature comparison for both models.

\begin{tabular}{l|c|c}
\hline Name & Approximated Layout & Detailed Layout \\
\hline \hline HF Die & $129.1^{\circ} \mathrm{C}$ & $128.2^{\circ} \mathrm{C}$ \\
\hline LF Die & $115.8^{\circ} \mathrm{C}$ & $115.6^{\circ} \mathrm{C}$ \\
\hline Magnetic Core & $121.4^{\circ} \mathrm{C}$ & $125.5^{\circ} \mathrm{C}$ \\
\hline
\end{tabular}

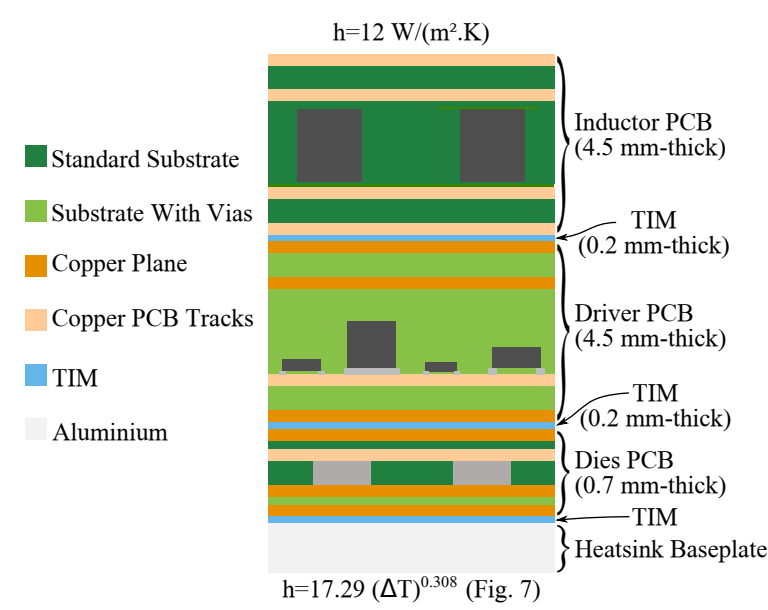

Figure 9: Stack-up used in the "Approximated layout" model.

$50 \mathrm{~W} /(\mathrm{m} \cdot \mathrm{K})[19]$, while the thermal conductivities in the $\mathrm{x}$-axis and $y$-axis remain the same. The thermal conductivity of the copper is $400 \mathrm{~W} /(\mathrm{m} \cdot \mathrm{K})$ in all directions for a plain layer. For a layer with only thin PCB tracks, the in-plane conductivities are considered to be those of the substrate layer $(0.3 \mathrm{~W} /(\mathrm{m} \cdot \mathrm{K}))$. The heatsink is only represented by its aluminium baseplate (4 mm-thick with $\lambda=237 \mathrm{~W} /(\mathrm{m} \cdot \mathrm{K})$ ). These anisotropic thermal conductivities are summarized in table I.

The boundary conditions are: a constant heat exchange coefficient on the top $\left(\mathrm{h}=12 \mathrm{~W} /\left(\mathrm{m}^{2} \mathrm{~K}\right)\right.$, calculated as the sum of natural air convection on a horizontal plane and heat radiation, for a $7 \times 7 \mathrm{~cm}$ surface at $125^{\circ} \mathrm{C}$ [20]); the heat exchange coefficient from Fig. 8 on the bottom; adiabatic condition on the sides; an ambient temperature of $60^{\circ} \mathrm{C}$. Heat is dissipated in the high and low frequency $\mathrm{SiC}$ dies and in the inductor. The power dissipated in each element is given in Tab. II (the calculation of these values is presented in [15]).

The structure of the model is presented in Fig. 9. Some results are presented in Fig. 10.

2) Detailed layout model : For this model, we directly import the detailed layout description (ODB++ file format generated from the PCB design software - Altium Designer) to build a 3D model in COMSOL. In order to simplify the model, the tracks and corresponding vias associated with the driver board are removed (the thermal vias of this board are kept). This model has 1500000 elements. The copper areas for the power and ground net are represented with their real layout. In this model, all thermal conductivities are isotropic.

As for the approximated layout mode, the baseplate of the heatsink and the different TIM layers are added to the model. All other simulation parameters (boundary conditions, material properties, etc.) are identical. Some results are given in Fig. 11.

\section{Simulation Results}

The results are listed in Tab. III. Both models predict that the junction temperature of the high frequency dies is close to $129^{\circ} \mathrm{C}$, while that of the low frequency dies is close to $115^{\circ} \mathrm{C}$ (in this converter topology, half of the semiconductor dies are switching at $180 \mathrm{kHz}$ while the second half only switches at the mains frequency, i.e. $50 \mathrm{~Hz}$ ). For the inductor, the detailed 


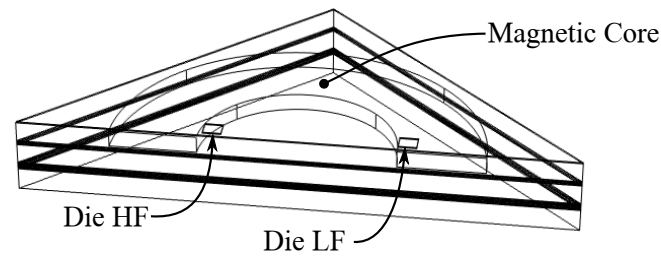

(a)

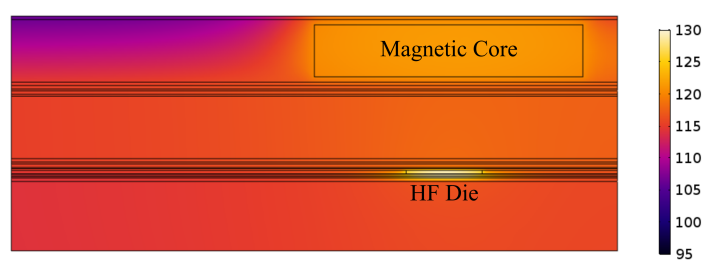

(b)

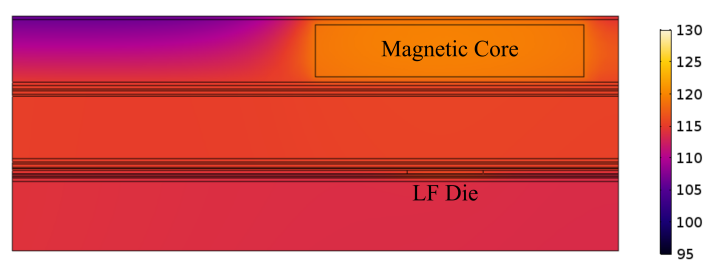

(c)

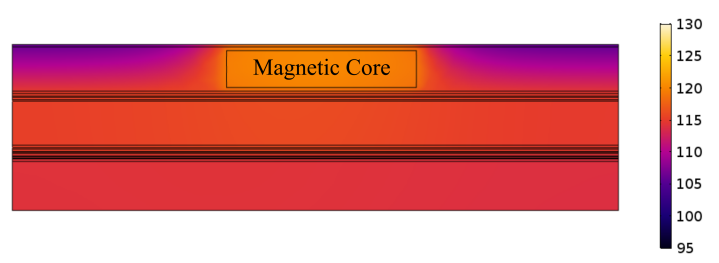

(d)

Figure 10: (a) Geometry of the "Approximated layout" model. A symmetry is used to reduce the computation time. Temperature plots over planes cutting through (b) the HF die and the center of the PCB, (c) the LF die and the center of the PCB and (d) the diagonal of the PCB.

layout model tends to predict slightly higher temperatures $\left(125.5^{\circ} \mathrm{C}\right.$ vs. $\left.121.4^{\circ} \mathrm{C}\right)$, probably because the driver board only offers high conductivity only where thermal vias could be inserted (the approximated layout model considers a uniform, relatively high conductivity in the z-axis over the entire driver board).

\section{THERMAL EXPERIMENT}

The objective of the thermal tests presented here is to assess the accuracy of the thermal models. In these tests, a set amount of power is dissipated by the $\mathrm{SiC}$ dies and the inductor, and the corresponding temperature inside the PCB stack is monitored using Resistance Temperature Detectors (RTDs) embedded in the PCBs. The experiment is performed in two stages: first a calibration step allows to set the dissipated power level for the inductor, for which it is difficult to directly measure the dissipated power. In a second stage, a complete PFC cell is submitted to the same power dissipation, and its internal temperature is recorded using the RTDs.

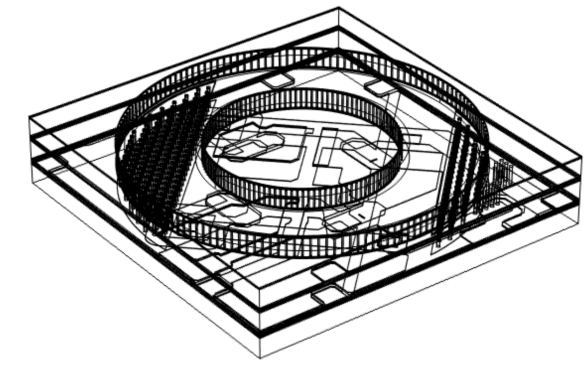

(a)

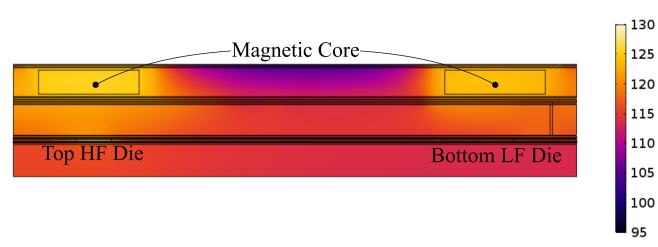

(b)

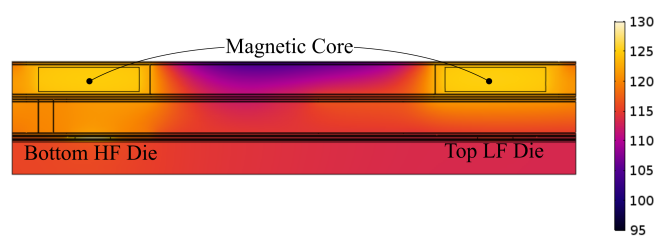

(c)

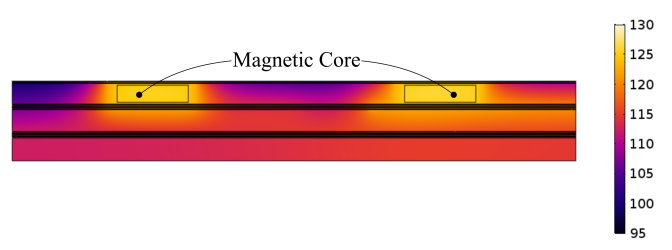

(d)

Figure 11: (a) Geometry of the "Detailed layout" model. Temperature plots over planes cutting through (c) the top HF die and the bottom LF die, (c) the bottom HF die and the top LF die and (c) a diagonal of the PCB.

\section{A. Calibration of the Dissipated Power}

The test circuit diagram used to dissipate power in the dies and inductor is presented in figure 12. Wires are soldered to the drain, source and gate of each die. The gate and the drain of each transistor are shorted together, so they can be considered as dipoles. This configuration allows to easily control the power dissipation in a transistor with a single power supply (in our case, two Source and Measure Units - references 2636 and 2602 - from Keithley, each having two independent channels as well as precision ammeters and voltmeters). Furthermore, as the transistors have a threshold voltage of around $3 \mathrm{~V}$, a realistic power dissipation can be achieved with with the moderate current level the sources can supply (3 A), which also has the advantage of limiting joule heating in the interconnects.

For the inductor, because (active) power dissipation is very small compared to the (mostly reactive) power involved, direct measurement of the dissipated electrical power would not be accurate. Instead, and indirect approach is chosen: calorimetry. 

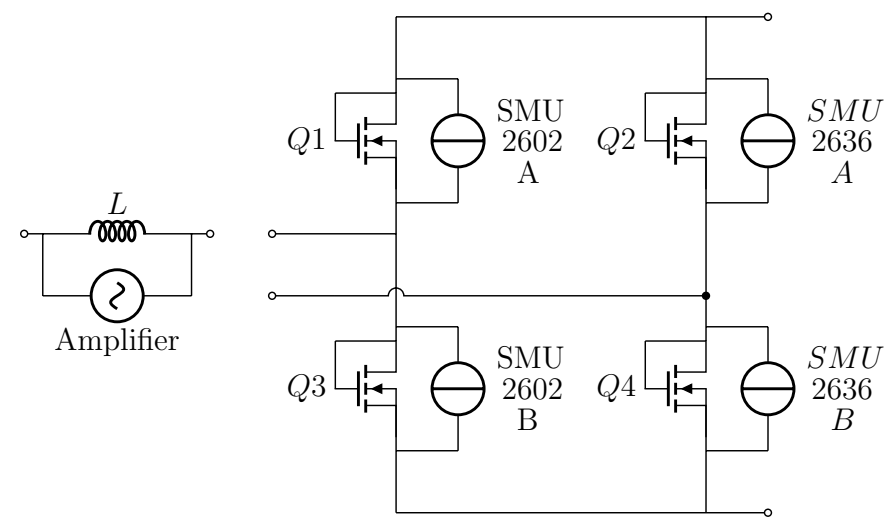

Figure 12: Circuit diagram of the thermal experiment.

Calorimetry is a simple and accurate way to measure losses. For that, the inductor is placed in a calorimeter (Figs. 14a and 14b) and supplied using an amplifier (AE Techron 7226) with a $70 \mathrm{kHz}$ sinewave. This frequency is chosen to obtain the highest possible power dissipation, considering the limitations of the amplifier. The calorimeter is composed of an enclosure which ideally should be adiabatic (in our case a Dewar jar with a plastic lid). A calibration resistor, the Device Under Test (DUT) and a RTD (connected to a Keithley 2700 precision multimeter) are placed in the enclosure, and immersed in a dielectric liquid (Novec 7500, 3M). This fluid is chosen because of its relatively low toxicity, its high boiling point $\left(128^{\circ} \mathrm{C}\right)$, its chemical and electrical compatibility with power electronics, and because it does not leave any residue after drying. A stirrer ensures a homogeneous temperature distribution of the bath.

The principle of this measurement setup is first to estimate the total heat capacity $\sum\left(m_{i} \cdot C_{i}\right)$ of the liquid bath and all the elements it contains by dissipating a set amount of energy using the calibration resistor and measuring the corresponding temperature rise of the bath. After this calibration phase, the DUT is operated for a set amount of time, and the corresponding temperature increase of the bath is measured again, this time to calculate the losses $P$. Figure 13 presents an example of the bath temperature during the losses measurement step. For both steps (calibration and measurement), eq (2) is used.

$$
P=\sum\left(m_{i} \cdot C_{i}\right) \cdot \frac{\Delta T}{t}
$$

The measurement error with this setup is estimated at $4.49 \%$, and mainly comes from the manual control of the heating time $( \pm 1 \mathrm{~s})$. This is considered sufficient in our case. In addition, note that calorimetric measurements are performed at a bath temperature of $\approx 30^{\circ} \mathrm{C}$, and that inductor losses will increase with temperature for a constant excitation signal. This effect, however, is not taken into account here.

\section{B. Measurement of the Internal temperature}

For the thermal test, a PFC cell is assembled and raised a few centimeters above the work surface to allow air flow in the heatsink placed on the bottom. The ambient temperature is $30^{\circ} \mathrm{C}$. The Board-to-Board interconnects are replaced with thin wires to connect the dies and inductor according to

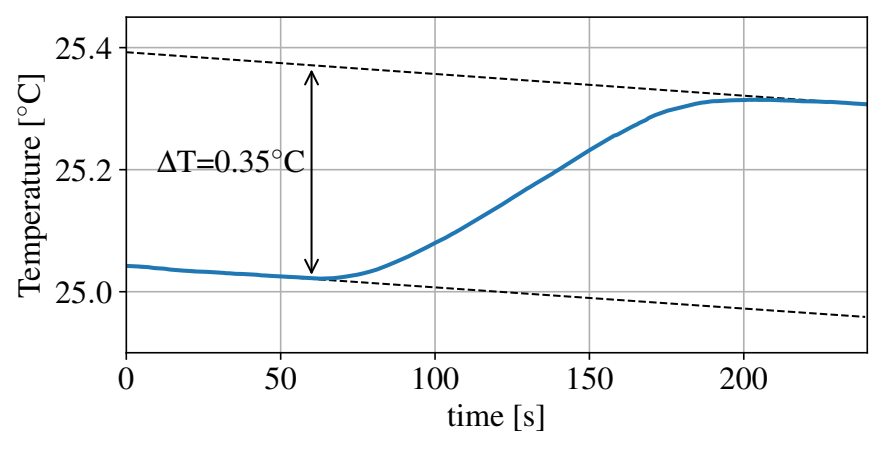

Figure 13: Bath temperature as a function of the time for the losses measurement step. The temperature difference between the two dashed lines corresponds to the temperature increase due to the operation of the DUT; the downward slope of the dashed lines is caused by heat leaks of the "adiabatic" enclosure.

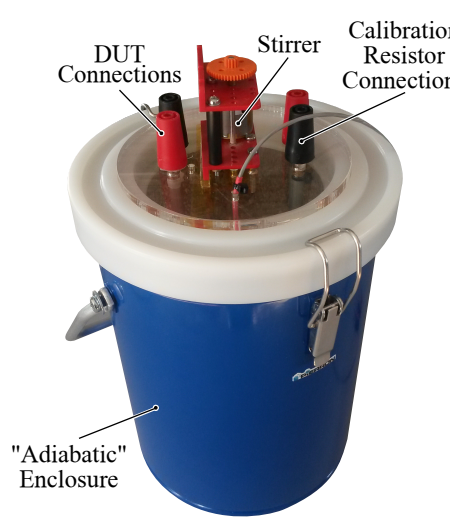

(a)

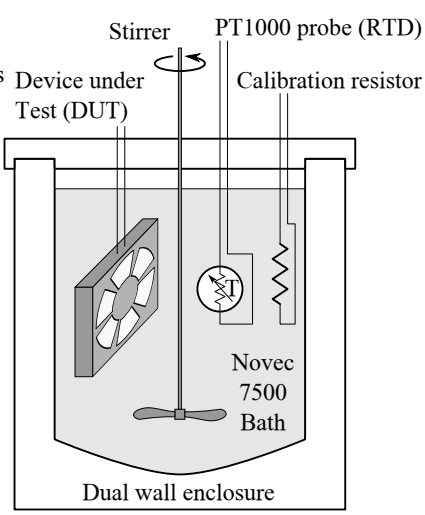

(b)
Figure 14: (a) Photograph and (b) diagram of the calorimeter. The (blue) dual wall enclosure has a diameter of $17 \mathrm{~cm}$ and a height of $22 \mathrm{~cm}$.

Fig. 12. The power sources (in particular the AC amplifier) are set as for the calibration phase. The RTDs are monitored using a Keithley 2700 multimeter, and the temperature values are recorded after a 30 min delay.

\section{Experimental Results}

The losses generated in each element are presented in Tab. IV. These values are lower than those estimated during the circuit design stage (Tab. II) due to limitations with the amplifier and the SMUs.

The temperatures inside a PFC cell are measured with 6 RTDs (PT1000 in this case). 4 RTDs are placed in the driver board, directly above the MOSFET dies. The 2 remaining RTDs are placed in the inductor PCB, one in the center and the other at the exterior of the inductor. The measured $\Delta T$ (temperature difference with the ambient temperature, measured at

Table IV: Losses generated in each power element of a PFC cell for the thermal tests.

\begin{tabular}{l|c}
\hline Dissipating element & Losses (W) \\
\hline \hline High Frequency Die (per die) & $4.00 \mathrm{~W}$ \\
\hline Low Frequency Die (per die) & $0.81 \mathrm{~W}$ \\
\hline Inductor & $2.00 \mathrm{~W}$ \\
\hline
\end{tabular}


Table V: Comparison of the temperature difference $(\Delta T)$ between the RTDs and the ambient obtained by experiment and simulation.

\begin{tabular}{c|c|c|c}
\hline RTD location & Experiment & $\begin{array}{c}\text { Approximated } \\
\text { Layout }\end{array}$ & $\begin{array}{c}\text { Imported } \\
\text { Layout }\end{array}$ \\
\hline \hline HF Die RTD & $\begin{array}{r}36.8^{\circ} \mathrm{C} \\
35.3^{\circ} \mathrm{C}\end{array}$ & $35.2^{\circ} \mathrm{C}$ & $\begin{array}{c}37.6^{\circ} \mathrm{C} \\
37.1^{\circ} \mathrm{C}\end{array}$ \\
\hline LF Die RTD & $33.8^{\circ} \mathrm{C}$ & $33.6^{\circ} \mathrm{C}$ & $34.1^{\circ} \mathrm{C}$ \\
$34^{\circ} \mathrm{C}$
\end{tabular}

$30^{\circ} \mathrm{C}$ ) are presented in Tab. V, along with the values calculated in the same conditions (same power dissipation, same ambient temperature) using the thermal models described above.

\section{DISCUSSION}

Overall, the results given in Tab. V show a very good agreement between experiment and simulation. In particular, the $\Delta T$ for the RTDs above the dies (LF and HF) falls inbetween the values predicted by both models. The maximum error for both models is $1.8^{\circ} \mathrm{C}$, which can be considered small in comparison with $\Delta T\left(>30^{\circ} \mathrm{C}\right)$.

For the RTDs located around the inductor, there is more difference between measured and simulated values (up to $4.9^{\circ} \mathrm{C}$ ). Furthermore, simulation results tend to systematically overestimate the temperature. This is particularly true for the "Approximated layout" model. This model does not consider the vias which form the inductor winding (Fig. 2). This may explain the higher temperature predictions. Also, for both models, a constant heat exchange coefficient value is considered on top of the PCB stack. This coefficient, however, is highly dependent on the temperature difference with the ambient.

The "Detailed layout" model tends to give results which are closer to the experiments, despite the simplifications which were performed to obtain an automated mesh. The computation time is $30 \mathrm{~min}$. An advantage of this model is the ease to describe the geometry of the board, as it is directly imported from Altium Designer. However, a manual alteration of the layout is still required to remove the finest tracks and some vias to make it compatible with automated meshing. The "Approximated layout" model tends to result in slightly larger errors, which remain acceptable for the dies. Its computation time is smaller ( $2 \mathrm{~min})$. An advantage of this model is that its generation can be automated (by calculating the copper fraction of each layer and the density of vias from the layout files).

In conclusion, both models generate satisfying results. The "Approximated layout" model is especially attractive. Indeed, it makes it possible to compute the accurate temperature distribution of a complex PCB stack in a few minutes. A possible refinement of this model is to consider subdivisions of each layers (for example $1 \mathrm{~cm}^{2}$ areas), each with their own thermal conductivities (to better match the layout of the boards). This would not require more elements in the mesh (for this model, the element size is dictated by the layer thickness, so that the element form factor remains acceptable), and would therefore not take longer to simulate.

Regarding the simulations presented in section IV-C (i.e. with the power dissipation level predicted by the circuit simulation and a worst case ambient temperature of $60^{\circ} \mathrm{C}$ ), the thermal models predict that all components remain at acceptable temperatures. The high frequency dies, which are the hottest points in the structure, are far from their quoted maximum junction temperature $\left(175^{\circ} \mathrm{C}\right)$. The gate driver IC, which has a maximum temperature of $125^{\circ} \mathrm{C}$, runs closer to its limits, with a calculated temperature of $123.8^{\circ} \mathrm{C}$. Running so close to the limit might be an issue, as it removes any safety margin for overload operation. A design iteration could be performed, based on the FEM simulations, to move the gate drivers to a milder place on the same PCB. In our case, in the absence of specifications regarding overload or other extreme operation cases, we consider that the current design meets the specifications.

\section{CONCLUSION}

A $3.3 \mathrm{~kW}$ AC/DC bidirectional converter stage has been implemented using 3 different boards: one for the semiconductor dies, one for the gate drive circuits, and one for the inductor. The manufacturing process has been described. To form a converter, all three boards were stacked on top of a heatsink.

Two modeling approaches were proposed for the stack: one is based on a simple approximation of each layer of the board, while the second considers a more accurate description of the board layout. Both models have been compared to experimental results, and show a good accuracy, especially for the dies). Regarding the inductor, the error is larger (up to $4.9^{\circ} \mathrm{C}$ difference between simulation and experiment).

Applying these models to the expected operating conditions of the converter show that the components temperatures should remain within specification. This is true despite the poor conductivity of the PCB substrate material and the thickness $(\approx 1 \mathrm{~cm})$ of the PCB stack. This demonstrates that with careful implementation, PCB embedding technology may be used for medium-power converters.

\section{REFERENCES}

[1] J. Millán, P. Godignon, X. Perpiñà, A. Pérez-Tomás, and J. Rebollo, "A Survey of Wide Bandgap Power Semiconductor Devices," IEEE transactions on Power Electronics, vol. 29, no. 5, pp. 2155-2163, May 2014.

[2] J. D. V. Wyk and F. C. Lee, "On a Future for Power Electronics," Journal of emerging and selected topics in power electronics, vol. 1, no. 2, pp. 59-72, 2013.

[3] J. W. Kolar, "What are the "Big CHALLENGES" in Power Electronics ?" in CIPS, 2014.

[4] R. Randoll, M. Asef, W. Wondrak, L. Böttcher, and A. Schletz, "Characteristics and aging of PCB embedded power electronics," Microelectronics Reliability, vol. 55, no. 9, pp. 1634 - 1639, 2015, proceedings of the 26th European Symposium on Reliability of Electron Devices, Failure Physics and Analysis. [Online]. Available: http://www.sciencedirect.com/science/article/pii/S002627141500164X

[5] C. Buttay, C. Martin, F. Morel, R. Caillaud, J. Le Leslé, R. Mrad, N. Degrenne, and S. Mollov, "Application of the PCB-Embedding Technology in Power Electronics - State of the Art and Proposed Development," in 3DPEIM, 2018. 
[6] A. Ostmann, "Evolution and future of embedding technology," in IMAPS/NMI workshop "disappearing die - embed your chips", 2016.

[7] AT\&S, "Embedded Device Packaging Ramping Up Next Generation SiP," JISSO European Council Seminar, 2011.

[8] D. Kearney, S. Kicin, E. Bianda, A. Krivda, and D. Bauman, "PCB Embedded Power Electronics for Low Voltage Applications," in CIPS, 2016, pp. 3-8.

[9] A. Alderman, L. Burgyan, B. Narveson, and E. Parker, "3-D Embedded Packaging Technology," IEEE Power Electronics Magazine, pp. 30-39, dec 2015.

[10] M. Ali, E. Labouré, F. Costa, and B. Revol, "Design of a hybrid integrated EMC filter for a DC-DC power converter," IEEE Transactions on Power Electronics, vol. 27, no. 11, pp. 4380-4390, 2012.

[11] R. Perrin, B. Allard, C. Buttay, N. Quentin, W. Zhang, R. Burgos, D. Boroyevich, P. Preciat, and D. Martineau, "2 MHz highdensity integrated power supply for gate driver in high-temperature applications," in Applied Power Electronics Conference and Exposition $(A P E C)$, Long Beach, United States, Mar. 2016. [Online]. Available: https://hal.archives-ouvertes.fr/hal-01372141

[12] E. Waffenschmidt, B. Ackermann, and J. a. Ferreira, "Design method and material technologies for passives in printed circuit board embedded circuits," IEEE Transactions on Power Electronics, vol. 20, no. 3, pp. 576-584, 2005.

[13] L. Codecasa, V. d'Alessandro, A. Magnani, and N. Rinaldi, "Compact dynamic modeling for fast simulation of nonlinear heat conduction in ultra-thin chip stacking technology," IEEE transactions on components, packaging and manufacturing technology, vol. 4, no. 11, nov 2014.

[14] A. Ziabari and A. Shakouri, "Fast thermal simulations of vertically integrated circuits ( $3 \mathrm{~d}$ ics) including thermal vias," in Proceedings of the $13^{\text {th }}$ IEEE ITHERM conference, 2012.

[15] J. Le Leslé, R. Caillaud, F. Morel, N. Degrenne, C. Buttay, R. Mrad, C. Vollaire, and S. Mollov, "Optimisation of an integrated bidirectional interleaved single-phase power factor corrector," in PCIM Europe 2018, International Exhibition and Conference for Power Electronics, Intelligent Motion, Renewable Energy and Energy Management, June 2018, pp. $1-8$.

[16] - "Optimum Design of a Single-Phase Power Pulsating Buffer (PPB) with PCB-integrated Inductor Technologies," in ICIT, 2018.

[17] R. Caillaud, C. Buttay, R. Mrad, J. Le Leslé, F. Morel, S. Mollov, and C. Martin, "Design , manufacturing and characterization of printed circuit board embedded inductors for power applications," in ICIT, 2018

[18] H. Perdomo Diaz and J. M. Codes Troyano. (2018) Heat Dissipation Challenge in Automotive High-Power Integrated Magnetics. [Online]. Available: lurl\{https://eepower.com/magnetics/ heat-dissipation-challenge-automotive-high-power-integrated-magnetics- 13

[19] G. Regnat, P.-O. Jeannin, G. Lefevre, J. Ewanchuk, D. Frey, S. Mollov, and J.-P. Ferrieux, "Silicon carbide power chip on chip module based on embedded die technology with paralleled dies," in Energy Conversion Congress and Exposition (ECCE), 2015 IEEE. IEEE, 2015, pp. 49134919.

[20] D. Annaratone, Engineering heat transfer. Springer Science \& Business Media, 2010.

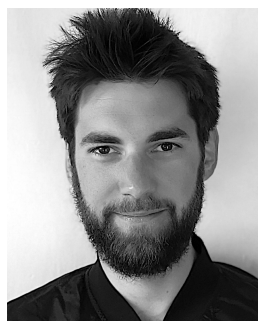

Rémy Caillaud, born 29/01/1991, received his engineer diploma and the M.S degree in engineering from École Centrale de Lyon, University of Lyon (France), in 2015. In 2019, he received his Ph.D degree in power electronics from INSA Lyon, University of Lyon for his research performed at the Ampère Laboratory in Lyon and Mitsubishi Electric R\&D Centre Europe in Rennes. He was part of the team awarded by the Semikron Innovation award in 2019 for the work done on the design of a Highly Integrated $3.3 \mathrm{~kW}$ AC/DC Converter Based on a Modular Approach. His main research interests include thermal management, PCB embedding technology and converter integration.

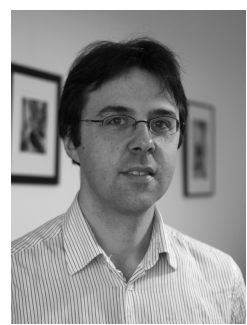

Cyril Buttay (M'04, SM'15) received the Engineer and $\mathrm{Ph} . \mathrm{D}$. degrees from the "Institut des Sciences Appliquées" (INSA) in Lyon, France, in 2001 and 2004 respectively. From 2005 to 2007 he was a research associate within the Electrical Machines and Drives research team of the University of Sheffield (UK) and the Power Electronics Machines and Control group of the university of Nottingham. Since 2008, he has been a scientist with the French Centre National de Recherche Scientifique (CNRS), working within the laboratoire Ampère, Lyon, France on the topic of packaging for power electronics, with a special focus on high temperature, high voltage or high density applications. He is currently a visiting scholar with the Center for Power Electronics Systems (CPES) at Virginia Tech, USA.

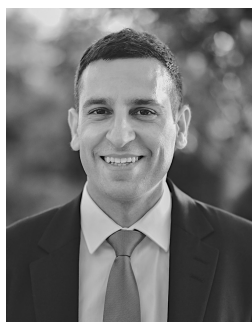

Roberto Mrad received his masters degree from the Lebanese University in Beirut and a PhD degree from the Ecole Centrale de Lyon in 2010 and 2014 respectively. Since 2014 he filled the position of power electronic packaging engineer at Mitsubishi Electric R\&D Center Europe. His main research interests are the multi-domain optimisation of power converters including the converter packaging, the design of magnetic components, EMC and thermal management. He received multiple IEEE best paper awards and holds multiple patents.

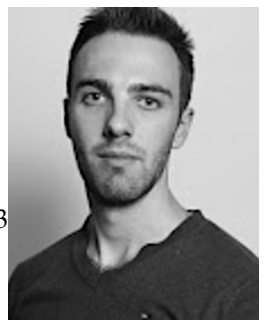

Johan Le Leslé received his engineer diploma and the M.S degree in electrical engineering from Polytech Nantes, University of Nantes, in 2015. In 2019, he received his $\mathrm{Ph} . \mathrm{D}$ degree in power electronics from the Ecole Centrale de Lyon, University of Lyon, for his research conducted with Mitsubishi Electric R\&D Centre Europe in Rennes and the Ampère Laboratory in Lyon. He was part of the team awarded by the Semikron Innovation award in 2019 for the work done on the design of a Highly Integrated $3.3 \mathrm{~kW}$ AC/DC Converter Based on a Modular Approach, which was a main part of his Ph.D activities. He is currently working as researcher for Mitsubishi Electric R\&D Centre Europe in Rennes. His main research interests include, multi-objective optimization, high efficiency, high power density and highly integrated converters.

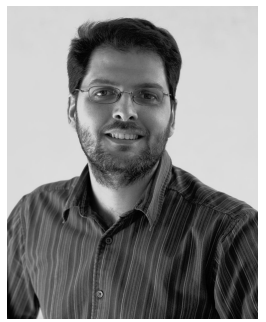

Florent Morel (S'06-M'07-SM'15) is an alumnus of École normale supérieure (ENS) de Cachan, France. In 2002, he obtained his "agrégation" in Electrical Engineering and he received an MSc in Electrical Engineering from INSA de Lyon, France in 2004. He pursued his $\mathrm{PhD}$ at INSA de Lyon on the control of electrical drives. He considered especially the association of a permanent magnet synchronous machine with a three-phase matrix converter. From 2007 to 2019, he was Associate Professor at École Centrale de Lyon and the Ampère Laboratory. He taught electrical engineering and power electronics. His research activities focused on power electronics converter design. He especially considered electromagnetic compatibility (EMC), use of silicon carbide devices, power supply of circuits with a high-voltage insulation and converters for high voltage grids (including railway). He is currently with Supergrid Institute, Villeurbanne, France. He is working on converters for HVDC applications. 


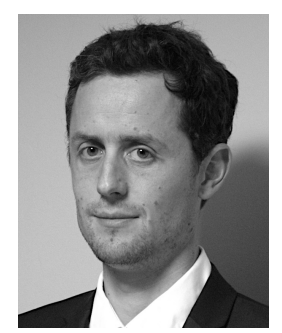

Nicolas Degrenne received M.Sc. and Ph.D. degrees in the field of Electrical Engineering from the "Institut National des Sciences appliquées" and from "École Centrale" Lyon, France, in 2008 and 2012, respectively. Since 2013 he has been working as a researcher in the fields of Integration, Reliability, Robustness, Diagnostics and Prognostics of Power Electronic Systems with Mitsubishi Electric R\&D Centre Europe, Rennes, France.

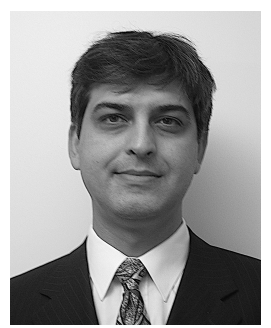

Stefan V. Mollov received the Dipl.Eng. degree from the Technical University of Gabrovo, Bulgaria, in 1995, and the Ph.D. degree from the University of Birmingham, U.K., in 1998.

From 2000, he was a Lecturer with the University of Birmingham, where his research activities focused on power electronics applications in the field of autonomous systems, aerospace electrical systems, and renewable energy sources and storage. In 2005, he joined ADETEL Group, France, as a Power Electronics Expert, working on transportation applications. In 2009, he joined THALES Avionics as a Power Supply Expert. Since 2013, he has been the Head of the Power Electronic Systems Division, Mitsubishi Electric R\&D Centre Europe, Rennes, France. His current interests lay in physics of failure, health and condition monitoring and highly integrated converters. 